N96- 15569

\title{
COMBUSTION OF A POLYMER (PMMA) SPHERE IN MICROGRAVITY
}

\author{
Jiann C. Yang and Anthony Hamins \\ Building and Fire Research Laboratory \\ National Institute of Standards and Technology \\ Gaithersburg, Maryland 20899
}

\section{Introduction and Objectives}

Since the advent of the manned-space exploration era, fire safety aboard space vehicles including space capsules, the Space Shuttle, and future manned permanent space-stations has been an important and challenging issue. An ideal way to alleviate a portion of the fire hazard is to eliminate completely the use of flammable materials or to replace them with materials with very high fire retardation properties. However, such an approach is not practical or technically feasible at present or in the immediate future. Materials (e.g., paper and plastics) that are flammable under certain conditions will still be used, and they will continue to pose a potential threat to the safety of the crew and the spacecraft. Therefore, the understanding of the combustion characteristics of such materials (e.g., ignitibility, flame spread rates, and burning rates) under different ambient conditions and in a reduced gravity environment is needed.

Polymer combustion is a highly complicated process where chemical reactions may occur not only in the gas phase, but also in the condensed phase as well as at the solid-gas interphase. The chemistry depends strongly on the coupling between the condensed phase and gas phase phenomena. For some polymers, additional complications arise due to the formation of char layers. For others, the behavior of the condensed phase involves swelling, bubbling, melting, sputtering, and multi-stage combustion (refs. 1-3). Some of these features bear resemblance to the phenomena observed in coal particle combustion. In addition to its relevance to spacecraft fire safety, the combustion of polymeric materials is related to many applications including solid and hybrid rocket propulsion, and of recent interest, waste incineration (refs. 4-6).

The burning rate is one of the most important parameters used to characterize the combustion of polymers. It has been used to rank the polymer flammability under the same experimental conditions and to evaluate various modes of inhibiting polymer flammability. The main objective of this work is to measure the burning rates of a polymeric material in low gravity. Because of inherent logistical diff:culties involved in microgravity experiments, it is impossible to examine a wide spectrum of polymeric materials. It is desirable to investigate a polymer whose combustion is less complicated, and yet will lead to a better understanding of the burning characteristics of other more complicated materials. Therefore, a typical non-charring polymer is selected for use in this experimental study. PMMA (polymethylmethacrylate) has been chosen because its thermo-physical properties are well characterized. Although the combustion of PMMA has been extensively studied in $1 \mathrm{G}$ experiments, only a limited amount of work has been conducted in low gravity (refs. 8-10).

A spherical sample geometry is chosen in this study because it is the simplest configuration in terms of the microgravity hardware design requirements. Furthermore, a burning PMMA sphere in microgravity 
represents a one-dimensional flame with overall combustion characteristics expected to be analogous to the combustion of a liquid fuel droplet (refs. 1-3), a field with many well-developed theories and models (ref. 7). However, differences can also be expected such as the flame-front standoff ratios and the condensed phase processes occurring during combustion.

\section{Research Approach}

It is anticipated that the ignition delay time (on the order of few seconds) of a PMMA sphere will be much longer than that of a liquid fuel droplet. Using a $1 \mathrm{G}$ burning rate constant $\left(2.76 \mathrm{~mm}^{2} / \mathrm{s}\right)$ from the literature (ref. 1) and assuming the " $\mathrm{d}^{2}$-law", the burning time of a PMMA sphere with a given initial diameter can be estimated. For a sphere with initial diameter of $1,3,5$, and $7 \mathrm{~mm}$, the calculated burning times are $0.36,3.3,9.1$, and $18 \mathrm{~s}$ respectively. Since the burning rate constant is expected to be lower under microgravity conditions, the above calculated values can only be regarded as lower bound estimates on the burning times in microgravity. Thus, the time available to carry out the experiments in both the 2.2 and $5.18 \mathrm{~s}$ NASA drop towers may not be adequate for the observation of complete burning histories (from ignition to burnout or extinction, if it occurs), even for a sphere with an initial diameter of $1 \mathrm{~mm}$. Therefore, the experiments will be conducted aboard a NASA DC-9 jet flying a parabolic trajectory in order to achieve longer durations (at least $20 \mathrm{~s}$ ) of a low gravity environment.

The intent of this work is to use a PMMA sphere which is large enough to allow good photographic resolution for observing the internal behavior of the condensed phase during combustion and at the same time to facilitate deployment of the sphere for the experiments. Two experimental approaches will be used in this study. The first involves the use of a suspended PMMA sphere and the second utilizes an unsupported PMMA sphere. These two techniques and the experimental procedure are discussed below.

A suspended sphere is obtained by using the set-up shown in Figure 1. A thermocouple (Pt/Pt-Rh) is stretched horizontally and heated locally near the thermocouple junction by resistive heating using two fine needles which are connected to four AA batteries and a potentiometer to regulate the current through the thermocouple wire. The sphere which is placed on a three-dimensional translation stage is then slowly positioned toward the heated wire. As the sphere approaches the heated portion of the thermocouple, part of it will become slightly soft so that the thermocouple junction is then easily inserted into the sphere. In this way, the thermocouple junction can be positioned at any location inside the sphere. The thermocouple has two functions; it is used to measure the temperature at one location inside the sphere and to suspend the sphere. Although local heating of the sphere during the embedding of the thermocouple may alter the properties of the sphere slightly, it is believed that the changes will be insignificant if the current through the thermocouple can be controlled in such a way that the temperature of the wire is barely sufficient to locally heat the sphere and if the heated area is very small, which can be achieved by using a fine thermocouple. We have successfully embedded 25,50 , and $75 \mu \mathrm{m}$ thermocouples. Although working with a $12.5 \mu \mathrm{m}$ thermocouple is possible, it has not been fruitful because of difficulties associated with the fragility of the wire. The aforementioned technique can also be extended to the suspension of two or more spheres (for studying the interaction between two burning spheres) provided that the wire is strong enough to support the weight of the spheres. Figures 2 and 3 show photographs of a nonburning suspended and two nonburning suspended spheres respectively. In the case of two or more spheres, only one sphere will have the thermocouple junction embedded in it.

Another technique for embedding a thermocouple has been tried but found to be unreliable. This method involved the dissolution of PMMA powder in toluene (a solvent). An initiator (benzoyl peroxide) was then added to the solution which was then cured in an oven until it became highly viscous. While the solution cured, a thermocouple junction was dipped in and out of the solution periodically and rotated slowly to form a nearly spherical bead. There were still many unresolved technical difficulties. Firstly, 
the positioning of the junction in the sphere was not possible. Secondly, the shape of the PMMA bead was not completely spherical. Lastly, bubbles were trapped or formed in the PMMA sphere during the fabrication process. Although two other techniques, injection molding and gluing of a sphere onto a quartz fiber, have been used in previous work, they have not been considered in this study based on the following two reasons. Use of injection molding is not practical because it is still very difficult to place a thermocouple junction at the desired location inside the sphere. Attaching a sphere onto a fiber precludes measurement of the temperature inside the sphere, although the technique is the simplest. Having compared the advantages and disadvantages among the four techniques, it is believed that for this study the embedding of a locally heated thermocouple junction in a PMMA sphere offers the best approach to form a suspended sphere. However, the present technique will not work on a thermosetting polymer.

The second experimental configuration that will be used in this work is an unsupported PMMA sphere. Figure 4 shows a (top view) schematic of the set-up which is currently under construction. The design is conceptually similar to the unsupported liquid droplet deployment system currently used in NASA drop tower experiments. Two opposing rods are used to support the sphere initially, and two ignition systems are used subsequently to ignite the sphere in a symmetrical manner. The supporting rods and ignition systems are mounted on four retracting mechanisms (also shown in Figure 4), which consist of springs, sliding blocks, and solenoids. Initially, the spring attached to the sliding block is stretched, the block is held in place by the solenoid plunger. The retracting action occurs when the solenoid is activated and its plunger retracts, and the spring pulls the sliding block away from its original position.

The current ignition method is adopted after other ignition techniques have been tried and found unsuccessful. One technique simply used a spark with very long discharge duration (more than $5 \mathrm{~s}$ ) to ignite the sphere. Ignition could not be achieved even when the sphere was preheated to a point just before melting of the sphere occurred. The second ignition method was to coat the sphere with a layer of ethanol, followed by spark ignition. This also proved to be unsuccessful because the spark simply ignited the liquid layer and burnt off the liquid fuel without ever igniting the polymer sphere. Ignition, itself, was always successful, using either a match or a small torch. The present ignition system is a modified version of a micro-torch that is evolved from the design of a cigarette lighter and a push-button igniter used on many gas $\mathrm{BBQ}$ grills. Figure 5 is a detailed drawing of the present ignition system. It consists of a small stainless steel fuel (liquid butane) reservoir, a needle valve to control the fuel flow, a miniature solenoid valve, a small jet nozzle, and a piezoceramic igniter element to generate a spark for igniting the fuel jet.

PMMA spheres with initial diameters of 3,5, and $7 \mathrm{~mm}$ will be studied. The suspended sphere or the unsupported sphere deployment system together with the ignition system will be housed inside a combustion chamber in which the ambient oxygen concentration can be varied in order to study the effect of oxygen concentration on the burning rate. The ambient oxygen concentration will be varied from $21 \%$ (by mole) in nitrogen (simulated air), to $45 \%$ and $70 \%$. A vitiated environment (19\% oxygen concentration) will also be tested. All the experiments will be conducted at room temperature and 0.101 $\mathrm{MPa}$.

The experimental procedure will involve the following steps. During normal flight paths, a suspended sphere, which is fabricated before flight, is placed in the combustion chamber. In the case of an unsupported sphere, the sphere is manually placed in the deployment mechanism. The ambient conditions in the combustion chamber are then set to the desired oxygen concentration. As soon as the change of G-level occurs, the sphere will be deployed (only for unsupported sphere experiments) and ignited. A high-speed movie camera (Locam) and a CCD camera will be used to record the evolution of the polymer sphere and flame diameter during combustion. The films will be analyzed frame-by-frame using an image 
analysis system. Temporal variation of temperatures inside the burning suspended sphere will also be recorded from the thermocouple using a data logger.

The experimental burning rate results will be compared to the predictions from existing liquid droplet combustion theories and polymer sphere burning models (refs. 1-3, 6).

\section{Results to date}

This work was started in August, 1994 although the funding was made available in mid June, 1994. The past six months were devoted solely to experimental hardware development. Several techniques for fabricating the thermocouple suspended sphere and for igniting a sphere have been attempted, and the most feasible ones have been identified. A set-up for suspending a PMMA sphere on a thermocouple has been constructed and is operational. The unsupported sphere deployment system is currently under construction, and the ignition system is currently being tested using suspended spheres in $1 \mathrm{G}$ conditions.

Some $1 \mathrm{G}$ suspended sphere experiments have been performed using commercial grade PMMA spheres with an initial diameter of $4.8 \mathrm{~mm}$. Preliminary observations can be summarized qualitatively as follows. There was an ignition delay of less than $5 \mathrm{~s}$ when a single ignition system was used. Upon ignition, the sphere softened, and internal bubbling occurred. The shape of the sphere started to deform, followed by dripping of the molten PMMA. Finally, the deformed, burning sphere fell off the suspended thermocouple wire in less than $10 \mathrm{~s}$ after ignition. Thermocouple measurements have not yet been made.

\section{$\underline{\text { Research Plans }}$}

The research plans for the rest of this FY and the next FY will be to finish the construction of the unsupported sphere deployment system, to fabricate the combustion chamber, to assemble the experimental package (rig), and to perform suspended sphere experiments using 3, 5, and $7 \mathrm{~mm}$ spheres and four ambient oxygen concentrations $(19 \%, 21 \%, 45 \%$, and $70 \%)$. It is anticipated that the unsupported sphere experiments will require a lot of fine tuning (e.g., coordination of timing of sphere deployment and ignition). Therefore, it is unlikely that a series of unsupported sphere experiments will be performed systematically although some tests are possible.

A flight proposal for using the Space Shuttle Middeck Glovebox (MGBX) Facility to perform a series of supported sphere experiments was submitted to NASA in February, 1995.

\section{References}

1. Essenhigh, R.H. and Dreier, W.L., "Combustion Behavior of Thermoplastic Polymer Spheres Burning in Quiescent Atmosphere of Air," Fuel, 48 (1969), pp. 330-342.

2. Raghunandan, B.N. and Mukunda, H.S., "Combustion of Polystyrene Spheres in Air," Fuel, 56 (1977), pp. 271-276.

3. Waibel, R.T. and Essenhigh, R.H., "Combustion of Thermoplastic Polymer Particles in Various Oxygen Atmospheres: Comparison of Theory and Experiment," Fourteenth Symposium (International) on Combustion, The Combustion Instinute, Pittsburgh, 1973, pp. 1413-1420.

4. Chung, S.L. and Tsang, S.M., "Soot Control During the Combustion of Polystyrene," J. Air Waste Manage. Assoc., 41 (1991), pp. 821-826.

5. Chung, S.L. and Lai, N.L., "Suppression of Soot by Metal Additives During the Combustion of Polystyrene," J. Air Waste Manage. Assoc., 42 (1992), pp. 1082-1088.

6. Panagiotou, T. and Levendis, Y., "A Study of Combustion Characteristics of PVC, Poly(styrene), Poly(ethylene), and Poly(propylene) Particles under High Heating Rates," Comb. \& Flame, 99 (1994), pp. 5374.

7. Williams, F.A., "Combustion Theory," Benjamin-Cummings, Menlo Park, CA, 1985.

8. Melikhov, A.S., Potyakin, V.I., Ryzhov, A.M., and Ivanov, B.A., "Limiting Polymer Combustion Regimes 
in the Absence of Free Convection," Comb. Expl. \& Shock Waves, 19 (1983), pp. 393-395.

9. Goldmeer, J.S., Urban, D.L., and T'ien, J.S., "An Experimental Study of Solid Diffusion Flame Extinction and Transition to Microgravity," Eastern States Section of the Combustion Institute, Fall Technical Meeting, Princeton University, Princeton, NJ, October 25-27, 1993, pp. 441-444.

10. Olson, S.L. and Hegde, U., "Imposed Radiation Effects on Flame Spread over Black PMMA in Low Gravity," Eastem States Section of the Combustion Institute, Fall Technical Meeting, Clearwater Beach, FL, December 5-7, 1994, pp.348-351.

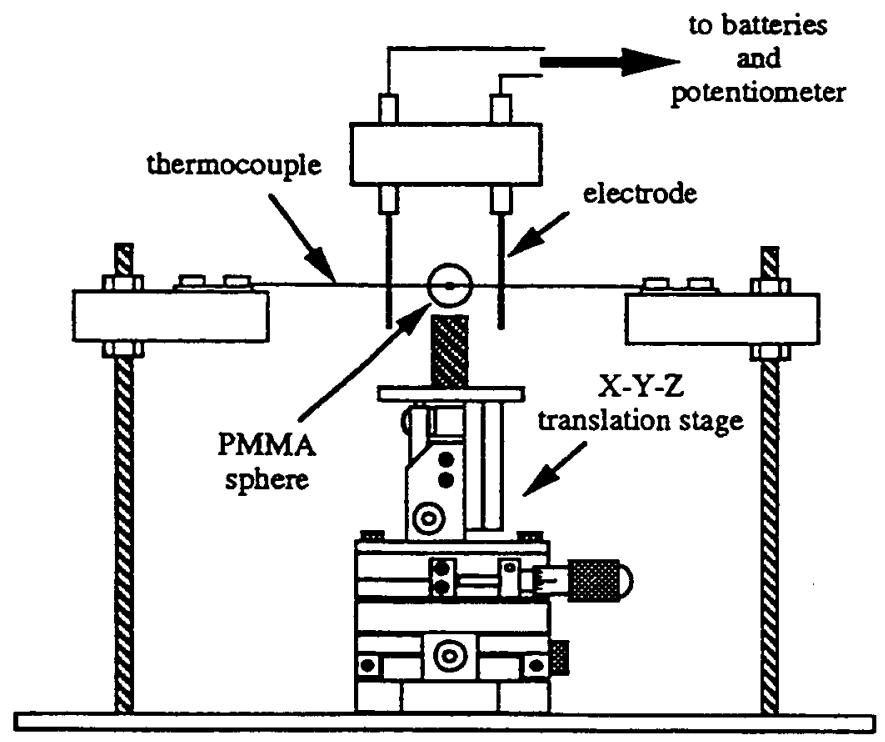

Figure 1.- Schematic of the suspended polymer sphere fabrication set-up.

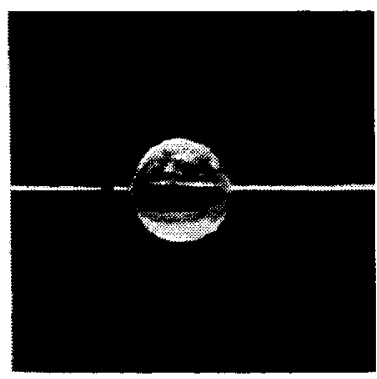

Figure 2.- Photograph of a suspended $4.8 \mathrm{~mm}$ PMMA sphere on a $75 \mu \mathrm{m}$ thermocouple.

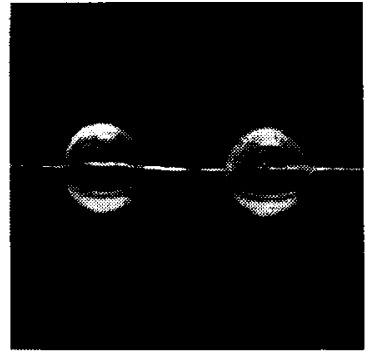

Figure 3. - Photograph of two suspended 4.8 mm PMMA spheres on a $75 \mu \mathrm{m}$ thermocouple. 


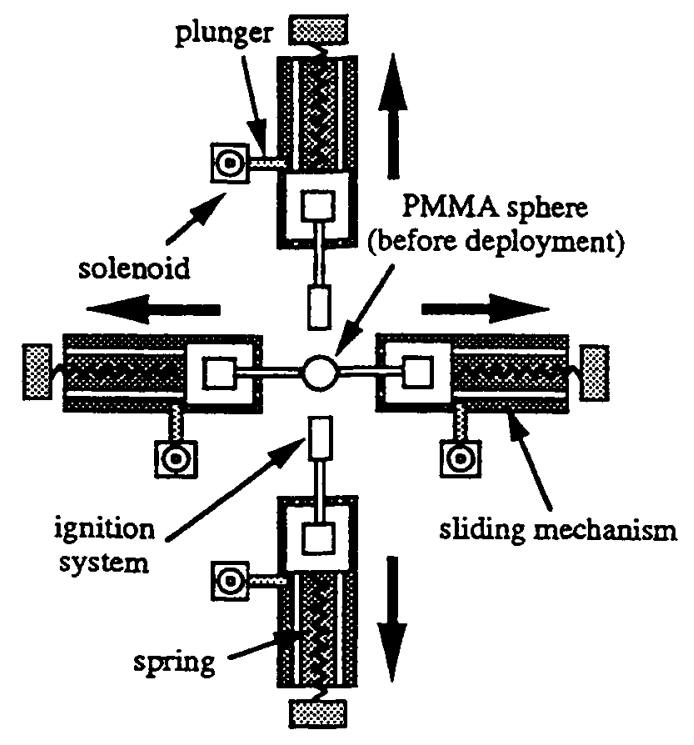

Figure 4.- Schematic of the unsupported sphere experimental set-up.

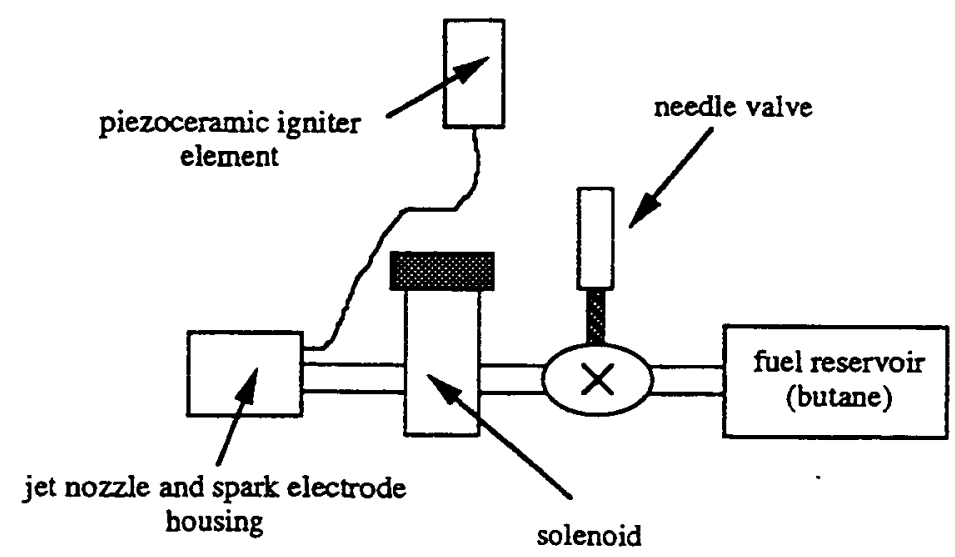

Figure 5.- Schematic of the ignition system. 